\title{
TENDENCIAS DE PUBLICACIÓN EN USTASALUD DESDE 2002 HASTA 2014: UN ANÁLISIS BIBLIOMÉTRICO
}

\author{
${ }^{1}$ Martha Juliana Rodríguez Gómez, ${ }^{2}$ José Fernando Pieruccini Arias, \\ ${ }^{3}$ Sonia Patricia Pieruccini Arias, ${ }^{4}$ Sonia Constanza Concha Sánchez
}

${ }^{1}$ Especialista en Odontopediatría y Ortodoncia Preventiva U. CES, magíster en Epidemiología U. Industrial de Santander, docente U. Santo Tomás, Colombia.

${ }^{2}$ Estudiante IX Semestre F. de Odontología U. Santo Tomás, Colombia.

${ }^{3}$ Odontóloga U. Santo Tomás, Colombia.

${ }^{4}$ Especialista en Educación y Comunicación para la Salud U. Industrial de Santander, magíster en Epidemiología U. Industrial de Santander, PhD (c) Salud Pública U. Nacional de Colombia, docente U. Santo Tomás, Colombia.

Docente responsable de correspondencia: Martha Juliana Rodríguez Gómez

Correo electrónico: marthajuro@mail.ustabuca.edu.co

\section{RESUMEN}

Objetivo: realizar un análisis bibliométrico de todos los artículos publicados en Ustasalud desde el segundo semestre de 2002 hasta el primero de 2014.

Materiales y métodos: se realizó un análisis bibliométrico descriptivo que incluyó la totalidad de artículos publicados en los 24 números que fueron revisados en físico. Se determinó el diseño del estudio, el tipo de artículo, su área temática, el número de autores, el género, la afiliación, y la actividad del primer autor. Se calcularon medidas de tendencia central y dispersión así como frecuencias y proporciones, una prueba t de Student o U. de Mann Whitney fue aplicada según la distribución de la variable. Se calculó el índice de productividad de Lotka y el índice de transitoriedad.

Resultados: se publicaron 170 artículos, el artículo original significó más de la mitad de la producción (62,9\%) seguido del reporte de caso $(17,1 \%)$. Los diseños de estudio más frecuentes fueron el de corte transversal $(52,3 \%)$ y el diseño experimental in vitro $(23,4 \%)$. Salud pública y epidemiología fueron los temas de interés predominantes con $56(32,9 \%)$ trabajos. La afiliación del primer autor mostró una participación interna del $77,6 \%$ y externa del $22,4 \%$; el autor con mayor número de publicaciones obtuvo un índice de Lotka de 1,4 (Concha SC), el índice de transitoriedad fue de 0,8 debido a que $84,7 \%$ de autores solo publicaron en una ocasión.

Conclusiones: la revista Ustasalud ha divulgado gran cantidad de estudios que en su mayoría corresponden a productos realizados por estudiantes y docentes vinculados con la Universidad Santo Tomás [Rodriguez MJ, Pieruccini JF, Pieruccini SP, Concha SC. Tendencias de publicación en Ustasalud desde 2002 hasta 2014: un análisis bibliométrico. Ustasalud. 2014; 13: 40 - 48]

Palabras clave: Bibliometría, odontología, artículo de revista.

\section{TRENDS IN USTASALUD JOURNAL FROM 2002 TO 2014: A BIBLIOMETRIC ANALYSIS}

Objective: to analyze all papers published in Ustasalud journal since mid 2002 to the first semester of 2014.

Methods: a descriptive bibliometric analysis including all articles published in the 24 issues of Ustasalud were reviewed. Study design, type of article, subject area, number of authors, and gender, affiliation, and activity of the first author were determined. Measures of central tendency and dispersion as well as frequencies and proportions were calculated; a Student's t test or Mann-Whitney U was applied according to the distribution of the variable. The productivity and isolation index were established.

Results: 170 papers were published, it was found that more than half of the production (62.9\%) was original papers followed by theme review articles (13.5\%). The most common study designs were cross-sectional (52.3\%) followed by experimental In vitro design (23.4\%). Public health and Epidemiology were the main themes of interest with 56 (32.9\%) papers. The affiliation of the first author showed that most authors (77.6\%) belonged to the Universidad Santo Tomás and $22.4 \%$ was affiliated with external institutions. The author with the highest number of publications had a productivity index of 1.4 (Concha SC), the isolation index was 0.8 , it meant that $84.7 \%$ of authors published only once in this journal.

Conclusiones: Ustasalud had published several studies, mostly written by teachers and students affiliated with Universidad Santo Tomás. [Rodriguez MJ, Pieruccini JF, Pieruccini S, Concha SC. Tendencias de publicación en Ustasalud durante doce años: un análisis bibliométrico.

Keywords: Bibliometrics, dentistry, epidemiologic studies. 


\section{INTRODUCCIÓN}

Hoy en día, las revistas científicas son consideradas una fuente de conocimiento esencial para la toma de decisiones basadas en la evidencia. El número de estas publicaciones ha aumentado considerablemente en todos los ámbitos. Por ejemplo, Colombia contaba con tres revistas odontológicas indexadas en el Índice Bibliográfico Nacional Publindex en julio de 2006, para junio de 2014, número que ascendió a siete. Este auge podría indicar un mayor interés por parte de los investigadores para divulgar su trabajo, con el fin de mostrar sus resultados, obtener satisfacción personal o reconocimiento, cumplir con las características de clasificación de investigadores emitidas por el Departamento Administrativo de Ciencia, Tecnología e Innovación (Colciencias) o conseguir puntos que mejoren su condición salarial, entre otros.

Es tal la cantidad de información que proporcionan los libros y las revistas científicas que se han desarrollado métodos para evaluarla mediante el uso de análisis estadísticos. A estos métodos se les ha dado el nombre de bibliometría, que etimológicamente se refiere a "libro" (biblio) y "medida" (metría). ${ }^{1}$ Según Yang y colaboradores, la evaluación de la literatura científica se realiza en tres pasos, el primero se encarga de recoger y valorar la evidencia disponible, en el segundo se evalúa la calidad de dicha evidencia y, en el tercer paso, se sintetiza la información para inferir sobre un tema en particular. ${ }^{2-4}$

Este trabajo se enfocó en el primer paso, es decir, en la recolección de los artículos publicados en Ustasa$l u d$, una de las revistas odontológicas colombianas que se encuentra indexada en Colciencias. Este es un paso importante si se tiene en cuenta que hasta el momento, no hay disponible en la literatura un análisis bibliométrico sobre los artículos publicados en alguna de las revistas odontológicas en Colombia.

Ustasalud es una publicación científica semestral vinculada con la Facultad de Odontología de la Universidad Santo Tomás en Bucaramanga, Colombia. El primer ejemplar apareció a finales de 2002 y se indexó en Publindex y Latindex en julio de 2006. Se ha destacado por la divulgación de artículos de investigación científica y tecnológica, artículos de revisión, revisiones de tema y reportes de caso. En estos doce años, no se ha realizado un estudio sobre los trabajos publicados, sus autores, las temáticas más frecuentes y las instituciones que han hecho parte de cada uno de los números, entre otros.

Por tanto, el objetivo de este estudio fue realizar un análisis bibliométrico de todos los artículos publicados en Ustasalud desde el volumen 1 número 1 (julio
- diciembre de 2002) hasta el volumen 13 número 1 (enero - junio de 2014) con el fin de valorar las tendencias que ha seguido la revista en los doce años que ha estado en circulación.

\section{MATERIALES Y MÉTODOS}

Se realizó un análisis bibliométrico descriptivo con todos los artículos publicados en Ustasalud desde el volumen 1 número 1 (julio - diciembre de 2002) hasta el volumen 13 número 1 (enero - junio de 2014), sin incluir el presente trabajo. De esta manera, se revisaron 170 artículos distribuidos en 24 revistas. Todos los ejemplares se obtuvieron en físico.

Como criterio de inclusión se tuvo en cuenta que el artículo fuese original, de revisión, revisión de tema, reporte de caso o serie de casos. Aspectos como el año, volumen, número del ejemplar, indexación, diseño de estudio, área temática, tipo de artículo, número de autores y de publicaciones por autor, género, afiliación institucional (institución, país) y nivel de escolaridad del primer autor fueron analizados.

La variable indexación se categorizó en indexada y no indexada en Publindex. Las categorías para identificar el diseño de estudio fueron reporte de caso, serie de caso, corte transversal, casos y controles, cohorte, ensayo clínico, ensayo comunitario, estudio cuasi experimental, revisión sistemática, experimental in vitro y otro. En esta última, se clasificaron los estudios de evaluación de tecnología diagnóstica y los realizados con animales en condiciones controladas.

Se manejaron las siguientes definiciones operativas para establecer el tipo de artículo: ${ }^{5}$

- Artículos originales: escritos que presentaban resultados derivados de proyectos originales de investigación. Su estructura constaba de cuatro apartados que eran Introducción, Metodología, Resultados y Discusión.

- Artículos de revisión: estudios realizados con el fin de dar una visión general sobre un tema que se caracteriza por tener un número de citas bibliográficas igual o superior a cincuenta.

- Revisión de tema: documento resultado de una revisión crítica y breve sobre un tema específico que cita menos de 50 referencias.

- Reporte de casos: trabajos cortos en el que se describe una experiencia de la práctica clínica. Un estudio serie de casos fue aquel que reportaba más de un caso clínico.

El área temática se clasificó según las especialidades odontológicas (cirugía oral y maxilofacial, endodoncia, odontopediatría, ortodoncia, patología oral, periodoncia y rehabilitación oral/materiales denta- 
les. Adicionalmente, se incluyeron como temáticas salud pública y epidemiología, odontología legal y forense y otra (diferente a las ya mencionadas).

Se determinó el número de autores participantes en todos los artículos publicados en los 24 ejemplares de la revista y además, se analizó el número de publicaciones por autor, es decir, la suma de todas las publicaciones de cada autor independiente de la posición de autoría que ocupara en el artículo.

La afiliación institucional se categorizó en interna, externa (nacional e internacional) y mixta. Interna se refirió a la producción intrainstitucional, es decir, cuando provenía de la Universidad Santo Tomás; externa nacional si los autores pertenecían a una institución colombiana, externa internacional si la institución no era colombiana y mixta si hubo una participación de autores con afiliaciones internas y externas.

Adicionalmente, se evaluó si el primer autor era estudiante de pregrado, estudiante de posgrado, docente o se dedicaba a otra actividad diferente.

\section{Procedimiento}

Se evaluaron los 170 artículos completos por tres investigadores que previamente realizaron una calibración interexaminador (coeficiente Kappa 0,9078 y 0,9692 ) e intra examinador (coeficiente Kappa 0,9692 para cada investigador). Posteriormente, estos fueron numerados y seleccionados aleatoriamente (http://www.alazar.info/generador-de-numeros-aleatorios-sin-repeticion) para ser distribuidos al azar. Se diseñó el instrumento para registrar las variables que iban a ser analizadas.

En la prueba piloto se estandarizó el instrumento, se estimó el tiempo de diligenciamiento y se estableció si se debía obtener más información. De esta manera, se concertó que era importante identificar el género y el nivel de escolaridad del primer autor.

Luego de recolectar la información, se realizó un control de calidad con el $20 \%$ de los instrumentos. De esta manera, 34 fueron escogidos al azar para valorar los datos registrados. Se encontraron ocho $(2,0 \%)$ inconsistencias en seis instrumentos que fueron inmediatamente corregidas.

\section{Procesamiento de la información}

Posteriormente, se realizó una doble digitación en bases creadas en Excel y se validaron mediante el software Epidata 3.1; los errores de digitación se compararon con los instrumentos para obtener una base completamente depurada que se exportó al paquete estadístico Stata I/C versión 12.0 para el análisis correspondiente. ${ }^{6-8}$

\section{Análisis estadístico}

Se obtuvieron frecuencias y proporciones para las variables cualitativas y medidas de tendencia central y dispersión para las cuantitativas. Se verificó la distribución normal de las variables cuantitativas y se aplicó la prueba t de Student o U. de Mann Whitney, según fuera apropiada para establecer si existía una diferencia estadísticamente significativa en el número de autores por artículo y en el contenido de los ejemplares no indexados en relación con los indexados. La prueba Chi cuadrado o test exacto de Fisher fue utilizada para valorar la relación entre la actividad realizada por el primer autor y el tipo de artículo publicado. Un valor de $\mathrm{p}<0,05$ fue considerado estadísticamente significativo.

Indicadores bibliométricos: se calcularon dos indicadores de productividad, el índice de productividad de Lotka y el índice de transitoriedad. El primero hace referencia al logaritmo del número de publicaciones por autor (IP $=\log \mathrm{N}$ ) y el segundo al cociente entre el número de autores con un trabajo y el número total de autores. ${ }^{9}$

Mediante el índice de productividad se clasificaron los autores en grandes productores, aquellos con diez trabajos o más (IP $\geq 1)$; productores intermedios los que tenían entre dos y nueve artículos $(0<\mathrm{IP}<1)$ y los transitorios, quienes habían escrito un solo trabajo $(\mathrm{IP}=0)$, es decir, eran autores transitorios los que solo aparecían en una ocasión. ${ }^{10}$

\section{Consideraciones éticas}

Esta investigación se clasificó como una Investigación sin riesgo, según la resolución 8430 de 1993, artículo 11 puesto que el trabajo realizado fue documental. ${ }^{11}$ La información recolectada es pública y además de encontrarse la colección de Ustasalud en fisico en las bibliotecas de algunas facultades de odontología del país y del exterior, también está disponible en el URL: http://www.ustabuca.edu.co/gpresenzia/vista/tpl/ustabmanga/revista-ustasalud-odontologia.html

\section{RESULTADOS}

En los 24 números de Ustasalud se publicaron 170 artículos científicos de los cuales $107(62,9 \%)$ correspondieron a artículos originales, $8(4,7 \%)$ artículos de revisión, 23 (13,5\%) revisiones de tema, 29 (17,1\%) reportes de caso y $3(1,8 \%)$ fueron clasificados como otros (Figura 1).

El promedio de artículos originales por número fue $4,5 \pm 0,8$, el año en que hubo una mayor publicación de estos fue 2013 con doce artículos. El diseño de estudio que predominó fue el corte transversal al encontrarse en $56(52,3 \%)$ de los artículos originales, seguido de $25(23,4 \%)$ estudios experimentales in 
vitro; el que menos se publicó fue casos y controles, puesto que solo se observó un $(0,9 \%)$ trabajo en el volumen 13 número 1 de Ustasalud. En la categoría de otro se identificaron tres $(2,8 \%)$ estudios de evaluación de tecnología diagnóstica y un $(0,9 \%)$ estudio en animales (Figura 2$)$.
En relación con el área temática que más divulgación ha tenido se encontró que esta fue la Salud Pública y Epidemiología con 56 (32,9\%) artículos. Le siguieron Endodoncia y Odontopediatría con 28 $(16,5 \%)$ y $17(10,0 \%)$, respectivamente.

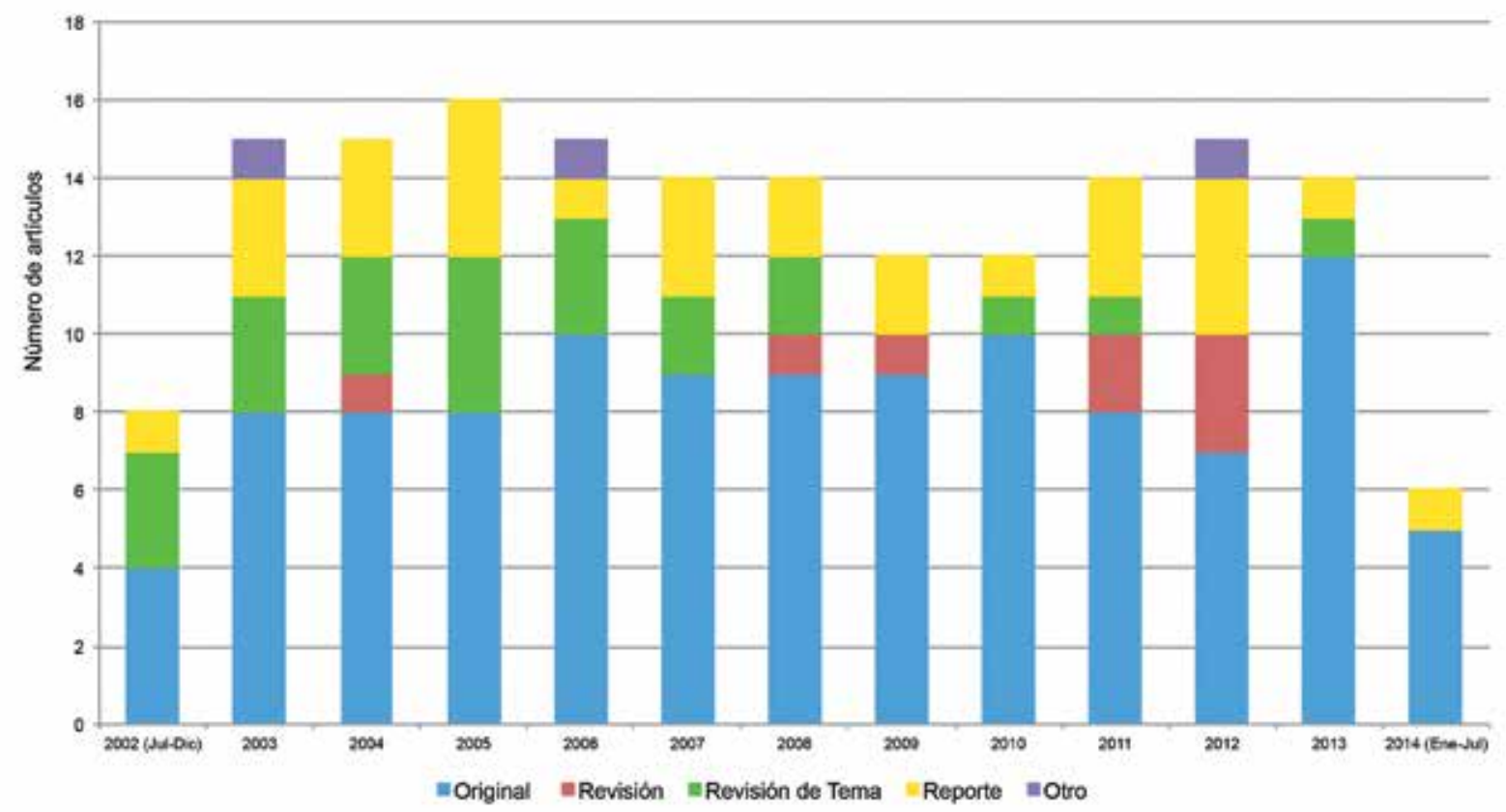

Figura 1. Distribución de los tipos de artículos publicados en Ustasalud desde el volumen 1 número 1 (julio - diciembre, 2002) hasta el volumen 13 número 1 (enero - junio de 2014)

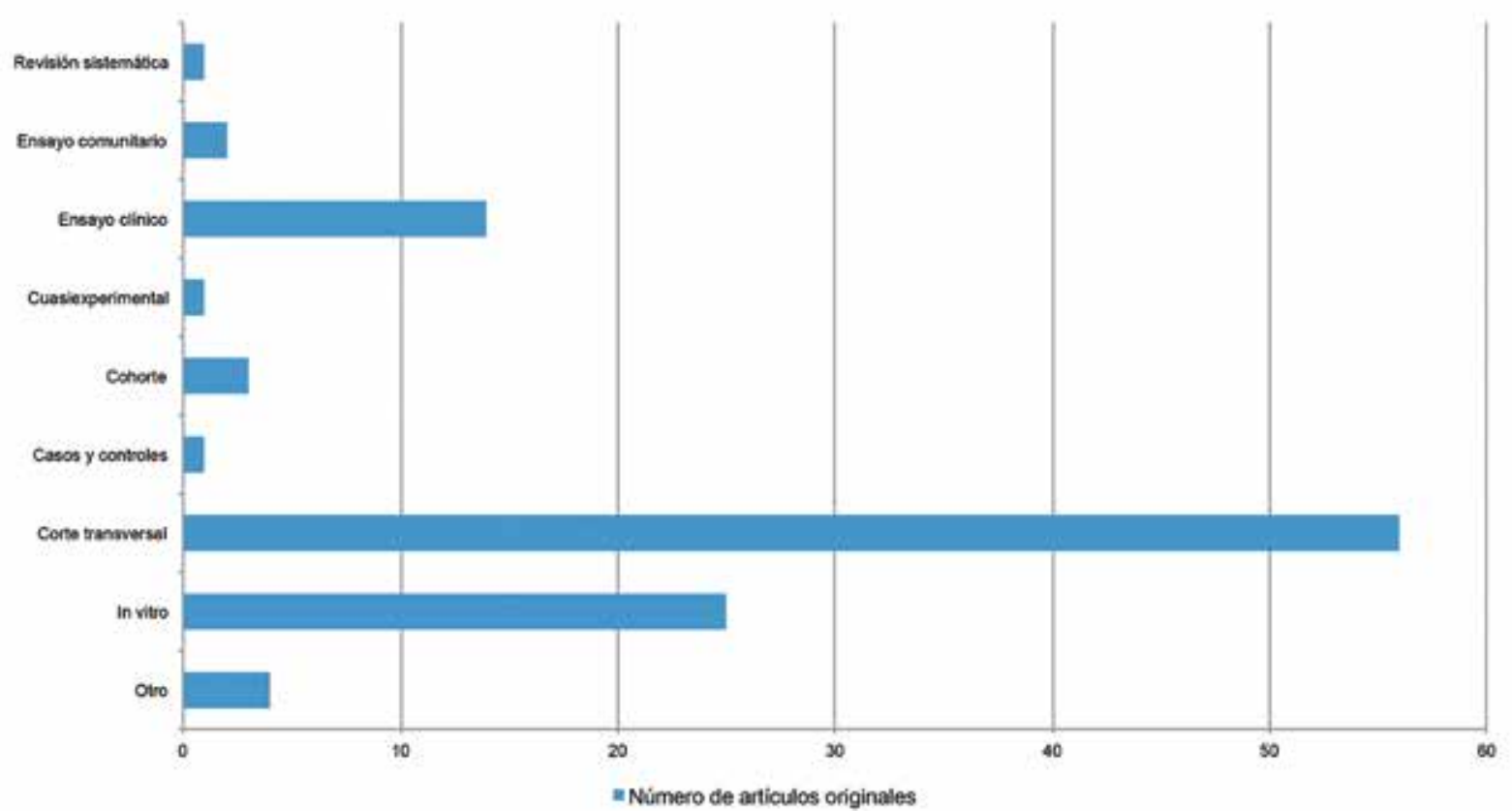

Figura 2. Distribución del diseño de estudio en los artículos originales publicados en Ustasalud desde el volumen 1 número 1 (julio - diciembre, 2002) hasta el volumen 13 número 1 (enero - junio de 2014) 
Quinientos sesenta y dos autores colaboraron en los 24 ejemplares durante los doce años, de estos $437(77,5 \%)$ participaron con artículos originales, $16(2,8 \%)$ con artículos de revisión, $48(8,5 \%)$ con revisiones de tema y $58(10,3 \%)$ con reportes de caso. Los tres $(0,5 \%)$ restantes contribuyeron con otro tipo de producción científica. El promedio de autores en un artículo original fue 4,1 \pm 1,6 con un rango que osciló entre 1 y 10 . Este valor disminuyó a 2,0 $\pm 1,2$ autores para el artículo de revisión y el reporte de caso. Al evaluar el género del primer autor de cada publicación se observó que $116(68,2 \%)$ eran mujeres (Tabla 1$)$.

Con respecto a la actividad que el primer autor desempeñaba, se encontró que más de la mitad de los colaboradores eran estudiantes de pregrado y posgrado, $48(28,2 \%)$ eran docentes universitarios y 24 $(14,1 \%)$ desarrollaban otra clase de labor. En cuanto a la afiliación institucional del primer autor, 132 $(77,6 \%)$ pertenecían a la Universidad Santo Tomás y $33(19,4 \%)$ trabajaban en diferentes universidades nacionales o extranjeras; cinco $(2,9 \%)$ no estaban vinculados con alguna entidad educativa (Tabla 2 ).

Tabla 1. Patrón de autoría de los artículos publicados en la revista Ustasalud de 2002 a 2014

\begin{tabular}{|c|c|c|c|c|c|c|c|c|}
\hline \multirow[b]{2}{*}{ Año } & \multicolumn{6}{|c|}{ Número de autores } & \multicolumn{2}{|c|}{ Género } \\
\hline & $\begin{array}{l}\text { Uno } \\
\text { n (\%) }\end{array}$ & $\begin{array}{c}\text { Dos } \\
\text { n (\%) }\end{array}$ & $\begin{array}{c}\text { Tres } \\
\mathrm{n}(\%)\end{array}$ & $\begin{array}{c}\text { Cuatro } \\
\mathrm{n}(\%)\end{array}$ & $\begin{array}{l}\text { Cinco } \\
\mathrm{n}(\%)\end{array}$ & $\begin{array}{c}>\text { cinco } \\
\mathrm{n}(\%)\end{array}$ & $\begin{array}{c}\text { Femenino } \\
\mathrm{n}(\%)\end{array}$ & $\begin{array}{c}\text { Masculino } \\
\text { n (\%) }\end{array}$ \\
\hline 2002 (Jul-dic) & - & $1(12,5)$ & $3(37,5)$ & $1(12,5)$ & $1(12,5)$ & $2(25,0)$ & $5(62,5)$ & $3(37,5)$ \\
\hline 2003 & $5(33,3)$ & $2(13,3)$ & $2(13,3)$ & $2(13,3)$ & $2(13,3)$ & $2(13,3)$ & $7(46,7)$ & $8(53,3)$ \\
\hline 2004 & $7(46,7)$ & $2(13,3)$ & $2(13,3)$ & $3(20,0)$ & - & $1(6,7)$ & $9(60,0)$ & $6(40,0)$ \\
\hline 2005 & $7(43,8)$ & $1(6,3)$ & $3(18,8)$ & $2(12,5)$ & $1(6,3)$ & $2(12,5)$ & $10(62,5)$ & $6(37,5)$ \\
\hline 2006 & $4(26,7)$ & $2(13,3)$ & $2(13,3)$ & $5(33,3)$ & $2(13,3)$ & - & $13(86,7)$ & $2(13,3)$ \\
\hline 2007 & $3(21,4)$ & $2(14,3)$ & $4(28,6)$ & $3(21,4)$ & $2(14,3)$ & - & $8(57,1)$ & $6(42,9)$ \\
\hline 2008 & $3(21,4)$ & $2(14,3)$ & $2(14,3)$ & $5(35,7)$ & $1(7,1)$ & $1(7,1)$ & $7(50,0)$ & $7(50,0)$ \\
\hline 2009 & $1(8,3)$ & $2(16,7)$ & $1(8,3)$ & $4(33,3)$ & $1(8,3)$ & $3(25,0)$ & $9(75,0)$ & $3(25,0)$ \\
\hline 2010 & - & $2(16,7)$ & - & $6(50,0)$ & $1(8,3)$ & $3(25,0)$ & $10(83,3)$ & $2(16,7)$ \\
\hline 2011 & $2(14,3)$ & $1(7,1)$ & $2(14,3)$ & $5(35,7)$ & $3(21,4)$ & $1(7,1)$ & $12(85,7)$ & $2(14,3)$ \\
\hline 2012 & $2(13,3)$ & $4(26,7)$ & $5(33,3)$ & $2(13,3)$ & - & $2(13,3)$ & $12(80,0)$ & $3(20)$ \\
\hline 2013 & $2(14,3)$ & $1(7,1)$ & $5(35,7)$ & $3(21,4)$ & $1(7,1)$ & $2(14,3)$ & $10(71,4)$ & $4(28,6)$ \\
\hline 2014 (Ene-jul) & - & $1(16,7)$ & - & $3(50,0)$ & $2(33,3)$ & - & $4(66,7)$ & $2(33,3)$ \\
\hline Total & $36(21,1)$ & $23(13,5)$ & $31(18,2)$ & $44(25,9)$ & $17(10,0)$ & $19(11,2)$ & $116(68,2)$ & $54(31,8)$ \\
\hline
\end{tabular}

Tabla 2. Distribución de los artículos evaluados según la institución de afiliación del primer autor, diferente a la Universidad Santo Tomás

\begin{tabular}{lc}
\hline Institución & n (\%) \\
\hline U. de Cartagena & $5(15,1)$ \\
U. de Antioquia & $4(12,1)$ \\
Fundación CIEO & $2(6,1)$ \\
U. Autónoma de México & $2(6,1)$ \\
U. CES & $2(6,1)$ \\
U. de Pamplona & $2(6,1)$ \\
U. de Sao Paulo & $2(6,1)$ \\
U. del Valle & $2(6,1)$ \\
U. Industrial de Santander & $2(6,1)$ \\
U. Nacional de Colombia & $2(6,1)$ \\
Fundación Cardiovascular & $1(3,0)$ \\
Fundación San Martín & $1(3,0)$ \\
NIHES & $1(3,0)$ \\
P. Universidad Javeriana, Cali & $1(3,0)$ \\
U. El Bosque & $1(3,0)$ \\
U. Estadual de Ponta Grossa & $1(3,0)$ \\
U. de Valencia & $1(3,0)$ \\
U. Stácio de Sá & $1(3,0)$ \\
\hline
\end{tabular}

44 
$\mathrm{Al}$ evaluar la relación entre la actividad que desempeñaba el primer autor y el tipo de artículo se encontró una diferencia estadísticamente significativa $(p<0,0001)$ que evidenció una proporción mayor de estudiantes de pregrado que publicaron un artículo original (Tabla 3). La afiliación institucional de los 42 estudiantes de pregrado correspondió a la Universidad Santo Tomás.

Así mismo, al analizar el número de autores por artículo, se observó una diferencia estadísticamente significativa $(p=0,0211)$ según la revista estuviera indexada o no. Es importante mencionar que 61 artículos hicieron parte de la publicación no indexada y 109 de la indexada desde julio de 2006 hasta la fecha. Al revisar el número de artículos originales y el número de artículos de revisión publicados no se encontró una diferencia estadísticamente significativa, pero si se halló al tener en cuenta la sumatoria de los dos tipos de artículos que Colciencias exige como una de sus condiciones para ser incluido en
Publindex $(p=0,0058)$ (Tabla 4).

Indicadores bibliométricos: se encontró que 62 autores habían escrito más de un artículo en Ustasalud. De estos, cuatro autores $(6,5 \%)$ fueron considerados como grandes productores, puesto que habían publicado 27, 16, 13 y 12 trabajos. De esta manera, el índice de productividad de Lotka para el autor con mayor número de artículos (Concha SC) fue 1,4, seguido de Aranzazu GC con un índice de 1,2, Camargo DM y Rodríguez MJ quienes obtuvieron el mismo valor $(\mathrm{IP}=1,1)$. Tres de ellos pertenecían a la Universidad Santo Tomás y uno estaba vinculado con la Universidad Industrial de Santander.

Los demás autores (58) publicaron entre 2 y 8 artículos por los que fueron clasificados como productores intermedios. De otra parte, 343 se catalogaron como autores transitorios, pues solo aparecían una sola vez en los 24 números. Es así como el índice de transitoriedad fue 0,8 .

Tabla 3. Relación entre la actividad que realizaba el primer autor y el tipo de artículo publicado (Ustasalud 2002 - 2014)

\begin{tabular}{lccccc}
\hline \multicolumn{1}{c}{ Variable } & $\begin{array}{c}\text { E. pregrado } \\
\mathbf{n}(\%)\end{array}$ & $\begin{array}{c}\text { E. posgrado } \\
\mathbf{n}(\%)\end{array}$ & $\begin{array}{c}\text { Docente } \\
\mathbf{n}(\%)\end{array}$ & $\begin{array}{c}\text { Otro } \\
\mathbf{n}(\%)\end{array}$ & $\mathbf{P}^{*}$ \\
\hline $\begin{array}{l}\text { Tipo de artículo } \\
\text { Artículos originales }\end{array}$ & $42(39,2)$ & $32(29,9)$ & $22(20,6)$ & $11(10,3)$ & $<0,0001$ \\
Artículos de revisión & $1(12,5)$ & $2(25,0)$ & $2(25,0)$ & $3(37,5)$ \\
Revisión de tema & $1(4,3)$ & $6(26,1)$ & $10(43,5)$ & $6(26,1)$ \\
Reportes de caso & $2(6,9)$ & $11(37,9)$ & $13(44,8)$ & $3(10,3)$ \\
\hline E. pregrado: estudiante de pregrado. E. posgrado: estudiante de posgrado. ${ }^{*}$ Test Exacto de Fisher. &
\end{tabular}

Tabla 4. Relación entre el promedio de autores por artículo publicado y tipo de artículo según la inclusión de la revista Ustasalud en Publindex

\begin{tabular}{lccc}
\hline \multicolumn{1}{c}{ Variable } & $\begin{array}{c}\text { No indexada } \\
\text { X } \pm \text { D.E. }\end{array}$ & $\begin{array}{c}\text { Indexada } \\
\text { X } \pm \text { D.E. }\end{array}$ & P \\
\hline Autores por artículo & $3,0 \pm 2,0$ & $3,5 \pm 1,6$ & $0,0211 \square$ \\
Artículos originales & $4,1 \pm 0,8$ & $4,6 \pm 0,8$ & $0,1707^{*}$ \\
Artículos de revisión & $0,1 \pm 0,4$ & $0,4 \pm 0,6$ & $0,2030 \square$ \\
Artículos originales + de revisión & $4,4 \pm 0,5$ & $5,2 \pm 0,7$ & $0,0058^{*}$ \\
\hline
\end{tabular}

X \pm D.E.: promedio \pm desviación estándar. * Prueba t de Student. $\square$ Prueba U. de Mann Whitney. 


\section{DISCUSIÓN}

Los resultados de este trabajo señalan que desde sus inicios, Ustasalud divulga una mayor proporción de artículos originales $(62,9 \%)$, seguido de reportes de caso $(17,1 \%)$ y revisiones de tema $(13,5 \%)$. Este hallazgo no es de sorprender si se tiene en cuenta que Colciencias exige un mayor número de artículos originales o de revisión publicados anualmente para acceder a una categorización en Publindex. Sin embargo, llama la atención la baja proporción de artículos de revisión $(4,7 \%)$.

Suárez encontró resultados similares al realizar un análisis bibliométrico de la revista Infectio (Revista de la Asociación Colombiana de Infectología) puesto que el 47,2\% de los artículos publicados correspondieron a artículos originales. ${ }^{9}$ Camps valoró cinco volúmenes de una revista de circulación trimestral (Universitas Médica) y encontró que la mayor proporción de trabajos eran artículos de revisión $(62,2 \%) .{ }^{12}$ Es importante mencionar que estas dos publicaciones colombianas se encuentran indexadas en $\mathrm{Pu}-$ blindex y por ende, deben cumplir con las características que se han descrito.

Algunos análisis bibliométricos de revistas internacionales como Serbian Dental Journal, Journal of Applied Oral Science y Jornal Brasileiro de Odontopediatría e Odontologia do Bebe también presentan un número importante de artículos originales sobre otro tipo de trabajos. ${ }^{13-15}$

En el presente trabajo se observó que las mujeres tuvieron una mayor participación al figurar como primer autor de los artículos analizados. Este dato difiere del obtenido por Estrada y colaboradores al evaluar la producción de la Revista Española de Salud Pública en la década 1991 - 2000, puesto que hallaron una mayor proporción de autores hombres $(56,5 \%) .{ }^{16} \mathrm{~A}$ este respecto, se debe advertir que en este estudio solo se tuvo en cuenta el género del primer autor y no el de sus colaboradores, como sí se hizo en el trabajo mencionado.

En relación con el área temática de los estudios de investigación publicados se observó que la revista se perfiló hacia los siguientes campos: Salud Pública y Epidemiología (32,9\%), Endodoncia (16.4\%) y Odontopediatría (10,0\%). Es posible que Salud Pública y Epidemiología sea el tema más frecuente debido a que la formación de los autores que más han publicado en la revista es esa. Esta información es comparable con la obtenida en el 2008 por Ferraz y colaboradores al evaluar la revista brasilera Journal of Applied Oral Science que evidenció que en tres años la endondoncia fue el área líder en la publicación con una proporción de 16,2\%; sin embargo, el listado continua con áreas como odontología restauradora $(12,9 \%)$ y materiales dentales $(10,1 \%)^{14}$ las cuales estuvieron por debajo de las tres áreas temáticas ya descritas para Ustasalud.

No es de extrañar que el diseño de estudio epidemiológico más frecuente haya sido el corte transversal dado sus múltiples ventajas como bajo costo y menor tiempo de ejecución si se compara con estudios como el de cohorte o el ensayo clínico. Poletto y Faraco también encontraron que entre los artículos originales, el estudio de corte transversal fue el más usual en el Jornal Brasileiro de Odontopediatría e Odontologia do Bebe. ${ }^{15}$ No obstante, Primo y colabores observaron que en el American Journal of Orthodontics and Dentofacial Orthopedics el 29,2\% de los artículos originales eran estudios de cohorte seguido de $26,6 \%$ de estudios de corte transversal en los números publicados en 1999, 2004 y 2009. Estos autores también revisaron la revista Dental Press Journal of Orthodontics y en esta, el 47,6\% eran estudios de corte transversal durante ese mismo periodo de tiempo. ${ }^{17}$

El patrón de autoría observado indica que la investigación generalmente se realiza en equipo, puesto que solo $36(21,1 \%)$ artículos fueron escritos por un único autor, los 134 (78,9\%) restantes tienen un rango que osciló entre dos y diez autores. García y colaboradores también hallaron similitud al encontrar en la evaluación la producción científica mexicana entre 1989 a $1996 .{ }^{18}$ Así mismo, 146 (85,9\%) de los artículos fueron escritos por personas vinculadas con instituciones de educación superior lo que podría sugerir que estas entidades incentivan a sus profesores y estudiantes a hacer investigación.

Es así como, $74(69,2 \%)$ artículos originales fueron escritos por estudiantes de pregrado y posgrado; de estos, 69 (93,2\%) estaban vinculados con la Universidad Santo Tomás. Estos datos demuestran que hoy en día, la revista divulga gran cantidad de investigaciones realizadas en la institución y que estas son el resultado de los trabajos de grado que los estudiantes deben cumplir como requisito para la obtención del diploma que los acredita como odontólogo o especialista.

En 2013, Colciencias divulgó el documento Indexación de revistas seriadas de ciencia, tecnología e innovación colombianas que establece nuevos requisitos para evaluar la calidad científica de las publicaciones en Colombia. Una de las condiciones más importantes es que la entidad editora solo puede publicar tres trabajos al año. ${ }^{19}$ Esta situación podría generar un impacto negativo en las revistas dependientes de las facultades de odontología del país, si se tiene en cuenta que gran parte de su producción es inter- 
na y corresponde a investigaciones realizadas por docentes y estudiantes de la institución.

Ustasalud fue indexada en Publindex en 2006, el hecho de haber encontrado una diferencia estadísticamente significativa entre el número de trabajos y de autores antes y después de estar indexada sugiere que la indexación creó gran interés en los investigadores, al entender posiblemente que su trabajo tendría así una mayor visibilidad. El índice de transitoriedad observado fue alto $(0,8)$; Suárez reportó un índice de transitoriedad para la revista Infectio (1995 a 2011) de 85,6\% que el autor también consideró elevado ${ }^{18}$.

Se podría pensar que una de las limitaciones de este trabajo fue no evaluar la calidad argumentativa o metodológica de los estudios publicados en Ustasalud, sin embargo, el propósito de esta investigación fue realizar un análisis descriptivo de los artículos publicados en cada uno de los 24 números desde el 2002 hasta el 2014 para identificar la tendencia que ha seguido la revista, áreas de interés o énfasis, autores, y determinar si el hecho de estar indexada en Publindex ha generado un cambio, ya sea en el número de artículos que se divulgan, número de autores o en la afiliación de estos. Al responder a esta potencial limitación, se propone que para futuros trabajos se analice tanto la calidad argumentativa, metodológica, las temáticas sobre las que se hace énfasis, así como los paradigmas a partir de los cuales se construye la investigación y, en este sentido, es importante retomar la experiencia desarrollada por Jaime Breilh en la que además de los aspectos ontológicos, se analizan lo metodológico y lo epistemológico con el propósito de alcanzar una mayor comprensión de la dinámica de la investigación odontológica que se reflejan en los trabajos publicados en la revista Ustasalud. ${ }^{20}$

Camps (2008) sugiere que es ideal combinar la estadística descriptiva y los indicadores bibliométricos con indicadores de impacto y juicio de expertos. ${ }^{21}$ A este respecto, es necesario mencionar que el factor de impacto (FI) se calcula gracias a las citaciones que reciben los artículos de las revistas, para lo cual es indispensable estar integrado en diferentes $\mathrm{Ba}$ ses Bibliográficas; hasta el momento, Ustasalud se encuentra indexada en Publindex, Latindex y en el Brazilian National Council for Scientific and Technologic Development (CAPES) y al no figurar en una base de datos como Scopus, no es posible calcular el FI.

Como se mencionó al inicio de este trabajo, este fue un primer paso con el fin de valorar la evidencia disponible en relación con la revista. Sin embargo, para tener un perfil más completo es necesario evaluar la calidad de los artículos y resumir la información publicada.

\section{REFERENCIAS}

1. Cronin B, Sugimoto CR. Beyond bibliometrics: Harnessing multidimensional indicators of scholarly impact. Cambridge: The MIT Press; 2014.

2. Yang S, Needleman H, Niederman R. A bibliometric analysis of the pediatric dental literature in MEDLINE. Pediatr Dent. 2001; 23: 415-418.

3. Assari $\mathrm{S}$, Ahmadyar M. Dental research in Iran: a bibliometric analysis of electronically available literature. Int Dent J. 2009; 59: 210-214.

4. Pritchard A. Statistical bibliography or bibliometrics. J Doc. 1969; 25: 348-349.

5. Colciencias. Documento guía. Servicio permanente de indexación de revistas de ciencia, tecnología e innovación colombianas 2010 [Mayo 30 de 2014]. URL disponible en: http://publindex.colciencias.gov.co:8084/publindex/docs/ informacionCompleta.pdf.

6. Microsoft Excel Corporation. Microsoft Excel. 2011: United States.

7. Epidata Association. Epidata Software 3.1. 2004: Denmark.

8. Corporation S. Statistical Software Stata I/C Release 12.0. 2011; College Station TX: United States.

9. Suárez JO. Análisis bibliométrico de la revista Infectio. Infectio. 2012; 16: 166-172.

10. Escorcia TA. El análisis bibliométrico como herramienta para el seguimiento de publicaciones científicas, tesis y trabajos de grado [Trabajo de Grado]. Bogotá: Pontificia Universidad Javeriana; 2008.

11. República de Colombia, Ministerio de Salud. Resolución No. 08430 de 4 de octubre de 1993.

12. Camps D. Estudio bibliométrico general de colaboración y consumo de la información en artículos originales de la revista Universitas Médica, periodo 2002 a 2006. Universitas Médica. 2007; 48: 358-365.

13. Jacimovic J, Zivkovic S. A bibliometric analysis of Serbian Dental Journal: 2002-2009. Serbian Dental Journal. 2010; 57: 76-81.

14. Ferraz VC, Amadei JRP, Santos CF. The evolution of the Journal of Applied Oral Science: a bibliometric analysis. J Appl Oral Sci. 2008; 16: 420-427.

15. Poletto VC, Faraco Junior IM. Bibliometric study of articles published in a Brazilian journal of pediatric dentistry. Braz Oral Res. 2010; 24: 83-38.

16. Estrada JM, Villar F, Péres C, Rebollo MJ. Estudio bibliométrico de los artículos originales de la Revista Española de Salud Pública (1991-2000). Parte segunda: productividad de los autores y procedencia institucional y geográfica. Rev Esp Salud Pública. 2003; 77: 333-346.

17. Primo NA, Gazzola VB, Primo BT, Tovo MF, Faraco IM. Bibliometric analysis of scientific articles published in Brazilian and international orthodontic journals over a 10-year period. Dental Press J Orthod. 2014; 19: 56-65.

18. García GA, García LA, Carreño MT, Maldonado AE, Rojas MdL. La productividad científica de la odontología en México. Revista ADM. 2010 67: 223-232. 
19. Departamento Administrativo de Ciencia Tecnología e Innovación. Indexación de revistas seriadas de ciencia, tecnología e innovación colombianas. Bogotá: Colciencias; 2013.

20. Breilh J. La determinación social de la salud como herramienta de transformación hacia una nueva salud pública (salud colectiva). Rev Fac Nac Salud Pública. 2013; 31: 1327.

21. Camps D. Limitaciones de los indicadores bibliométricos en la evaluación de la actividad científica biomédica. Colomb Med. 2008; 39: 74-79.

Correo electrónico de los autores:

Martha Juliana Rodríguez Gómez: marthajuro@mail.ustabuca.edu.co José Fernando Pieruccini Arias: josepieru@hotmail.com Sonia Patricia Pieruccini Arias: soniapieruccini@gmail.com Sonia Constanza Concha Sánchez: sococosa@yahoo.com 